\title{
Avaliação de Derivados Poliméricos Intercalados em Montmorilonita Organofilica na Preparação de Novos Materiais de Uso Farmacêutico
}

\author{
Camila B. Dornelas \\ Instituto de Macromoléculas Profa Eloisa Mano, UFRJ/Faculdade de Farmácia, UFAL \\ Daniel K. Resende, Helvecio V. A. Rocha, Ailton S. Gomes, Maria Inês B. Tavares \\ Instituto de Macromoléculas Profa Eloisa Mano, UFRJ
}

\author{
Sandra S. S. Coutinho, Lucio M. Cabral \\ Faculdade de Farmácia, UFRJ
}

Resumo: A nanotecnologia está relacionada às estruturas, propriedades e processos envolvendo materiais com dimensões numa escala nanométrica. O conceito de nanotecnologia em aplicações farmacêuticas, mais especificamente na liberação de fármacos, está baseado na produção de nanopartículas que contêm moléculas do fármaco a serem depositadas exclusivamente no órgão ou tecido alvo com a vantagem de serem inativas. Neste contexto, o presente trabalho busca estudar a utilização de uma organoargila, previamente modificada com octadecilamina, viscogel B8, como matriz de liberação controlada de fármacos por meio da reação de intercalação desta argila com PEG 6000 e MEG, comparando-se os resultados obtidos com um derivado previamente obtido da intercalação desta argila com PVP K-30. Estes materiais têm como diferencial a reunião de diferentes aspectos de funcionalidade, possibilitando se obter excipientes de liberação modificada de propriedades mistas.

Palavras-chave: Nanotecnologia, montmorilonita, MEG, PEG, PVP, liberação controlada.

\section{Evaluation of Use of Organophilic Montmorillonite and its Polymeric Intercalated Derivatives in Preparation of New Pharmaceutical Materials}

\begin{abstract}
Nanotechnology is related to structures, properties and processes involving materials in a nanometer dimension. This is widely used for pharmaceutical applications, more specifically in drugs' release. In this context, the present work studies the use of organophilic montmorillonite (OMMT), viscogel B8, as matrix of controlled release of drugs. New polymer-layered silicate nanocomposites (PLN) of MEG, PEG 6000 and OMMT were prepared and compared with PVP K-30-OMMT.
\end{abstract}

Keywords: Nanotechnology, montmorillonite, $M E G, P E G, P V P$, controlled release.

\section{Introdução}

$\mathrm{O}$ uso de produtos de origem natural como carreadores de fármacos apresenta-se como uma das mais atraentes e promissoras alternativas tecnológicas atuais, visto a maior biocompatibilidade destas substâncias em comparação aos materiais sintéticos, aliado a um menor custo de preparação. Dentre os novos sistemas terapêuticos mais investigados na atualidade, destacam-se os de dimensões nanométricas, os quais apresentam como vantagens principais a possibilidade de uma vetorização a nível celular ou tecidual do ativo neles contido, inclusive em termos temporais, proporcionando uma maior eficácia terapêutica e menores efeitos colaterais $^{[1]}$ quando comparados aos materiais poliméricos tradicionais. $\mathrm{O}$ termo nanomaterial faz referência a estruturas e propriedades envolvendo dimensões numa escala de 1 a $300 \mathrm{~nm}$, valores estes considerados elevados quando comparados a moléculas simples, porém pequenos em relação ao comprimento de onda da luz visível ${ }^{[2]}$. Todavia, a grande maioria das técnicas de preparação dos nanosistemas terapêuticos usuais se mostra extremamente custosa e com baixa probabilidade de escalonamento para fins industriais. Direciona-se, desta forma, a investigação do uso de nanomateriais pré-formados como alternativa para novos sistemas terapêuticos. Dos nanomateriais pré-formados disponíveis, destacam-se os silicatos lamelares, dentre eles, a montmorilonita sódica (principal constituinte da bentonita) e seus derivados intercalados. Estas argilas são filossilicatos lamelares do tipo 2:1, por apresentarem em sua estrutura básica dois octaedros de alumínio e magnésio em combinação com um tetraedro de sílica ${ }^{[3]}$. Substituições isomórficas de silício $\mathrm{Si}^{+4}$ por $\mathrm{Al}^{+3}$ no tetraedro de sílica e de $\mathrm{Al}^{+3}$ por $\mathrm{Mg}^{+2}$ no octaedro causam um excesso de cargas negativas dentro das lamelas da montmorilonita, as quais são contrabalanceadas em solução por cátions como $\mathrm{Ca}^{++} \mathrm{e} \mathrm{Na}^{+}$situados entre as mesmas ${ }^{[4]}$. Além destes cátions, substâncias

Autor para correspondência: Camila B. Dornelas, Universidade Federal de Alagoas, UFAL, ESENFAR, Faculdade de Farmácia, Campus A. C. Simões, Av. Lourival Melo Mota, s/n, Tabuleiro do Martins, CEP: 57072-970, Maceió, AL, Brasil. E-mail: dornelascb@yahoo.com.br 
positivamente carregadas, como íons alquilamônio ${ }^{[5]}$, podem ser inseridos no interior das lamelas, gerando um derivado organofílico, ou organoargila, de maior espaço interlamelar e passível de alteração de estrutura e propriedades. Dessa forma, ocorre uma diminuição da energia da superfície da argila de tal modo que materiais poliméricos tradicionalmente utilizados como matrizes hidrofílicas de liberação modificada podem ser intercalados entre as lamelas ${ }^{[6,7]}$, ou seja, um novo nanomaterial com combinadas características de intumescimento e lipofilicidade, extremamente útil em sistemas de liberação prolongada de fármacos.

Neste trabalho, investigou-se a utilização da montmorilonita organofílica, modificada com íons alquilamônio com cadeia de dezoito átomos de carbono (octadecilamina), como substrato para a reação de intercalação com poli(etileno glicol) (PEG) 6000 e monoestearato de glicerila (MEG), de forma a se obter excipientes de liberação modificada de propriedades mistas, hidrofílica/lipofílica. Os resultados obtidos foram, ainda, comparados com uma nova matriz derivada, com sucesso, da intercalação desta argila com a polivinilpirrolidona (PVP) K-30 $3{ }^{[8]}$.

\section{Experimental}

\section{Materiais}

Viscogel B8® (Bentec); dapsona (Gemini Exports); estearato de magnésio (Farmos); lactose, Cellactose 80 (Meggle); monoestearato de glicerila, MEG (Farmos); poli(etileno glicol), PEG 6000 (Sigma); polivinilpirrolidona, PVP K-30 (Sigma). Diclorometano (Tedia); tolueno (Tedia). Pacote estatístico STATISTICA (Stat Soft Company - USA). Em todas as soluções preparadas, utilizadas para a quantificação dos ativos por espectroscopia de ultravioleta e nos ensaios de dissolução, utilizou-se água recém destilada. Suco gástrico simulado (SGS) foi preparado conforme Farmacopéia Americana $30^{[9]}$.

\section{Preparo de nanocompósitos}

A técnica de preparação de nanocompósitos consistiu na solubilização do polímero no solvente de escolha e posterior adição de argila intercalada com sal de octadecilamina, de nome comercial viscogel B8, mantendo o sistema sob agitação.

Nos ensaios realizados, foram avaliados os efeitos da variação do tempo (15, 30, 45 minutos, 1, 24, 48 e 72 horas) e da temperatura (ambiente e sob refluxo) na reação. O produto das reações foi levado à filtração em papel de filtro. Ao material sólido resultante seco procedeu-se a pulverização em micro moinho de bolas (Aldrich). O cálculo do rendimento foi estimado por gravimetria.

\section{Caracterização}

\section{Análise por infravermelho (FTIR)}

Foram feitas análises dos nanocompósitos obtidos preparando-se pastilhas de $\mathrm{KBr}$ com amostras de cada produto nas melhores reações, sendo estas realizadas em espectrofotômetro FT-IR Perkin Elmer.

\section{Difração de raio $X$ (DRX)}

Foi utilizado um Difratômetro de raio X Rigaku, modelo Miniflex, operado a $40 \mathrm{kV}$ e $30 \mathrm{~mA}, 0,05$, em temperatura ambiente. A radiação $\mathrm{CuK}_{\alpha}$ foi utilizada como fonte dos raio $\mathrm{X}$, com comprimento de onda de $0,15418 \mathrm{~nm}$. Para obtenção do valor de espaçamento interlamelar foi realizado tratamento matemático e aplicação da equação de Bragg. Além das amostras reacionais, foram preparadas também as respectivas misturas físicas dos materiais utilizados nas reações. O viscogel B8 puro foi utilizado para fins comparativos, a fim de corroborar a formação de nanocompósitos. Ainda, foi feita uma reação do viscogel B8 com solvente apenas ("branco") para avaliar um possível efeito do solvente em sua estrutura ${ }^{[10]}$.

\section{Análise termogravimétrica (TGA)}

As amostras foram conduzidas em analisador termogravimétrico Perkin Elmer - TGA 7, com um suporte de alumínio em fluxo de nitrogênio com vazão de $50 \mathrm{~mL} / \mathrm{min}$ com uma taxa de aquecimento de $10{ }^{\circ} \mathrm{C} / \mathrm{min}$ de 100 a $850{ }^{\circ} \mathrm{C}$.

\section{Análise calorimétrica de varredura (DSC)}

As amostras foram analisadas em calorímetro de varredura diferencial Perkin Elmer - DSC 7, em fluxo de nitrogênio com vazão de $22,5 \mathrm{~mL} / \mathrm{min}$, em varredura de temperatura ascendente, entre 0 a $150{ }^{\circ} \mathrm{C}$, com taxa de aquecimento de $10{ }^{\circ} \mathrm{C} / \mathrm{min}$.

\section{Teste de determinação de toxicidade a $10 \mathrm{~g} / \mathrm{kg}$ de peso corpóreo}

Em 01 (um) grupo de 10 (dez) camundongos suíços, pesando $20 \mathrm{~g}( \pm 2 \mathrm{~g})$ foi administrada por via oral, através de uma cânula, uma dose do produto viscogel B8 de $10 \mathrm{~g} / \mathrm{kg}$ de peso corporal do animal diluída com água em $300 \mu \mathrm{L}$. Os animais foram observados durante 14 dias. Foi usado grupo controle. Os ensaios foram realizados segundo compêndio oficial ${ }^{[11]}$.

\section{Análise por cromatografia gasosa (CG)}

Foram preparadas soluções de concentração $5 \mathrm{mg} / \mathrm{mL}$ usando diclorometano como solvente para as amostras de MEG, octadecilamina, e sobrenadante (em duplicata), vindo do processo de filtração no processo de preparo do nanocompósito viscogel B8-MEG após evaporação do solvente, representado pelas amostras 1 e 2 . A coluna capilar usada, injetor e as condições estabelecidas para cada um deles no teste encontram-se nas Tabelas 1 e 2, respectivamente, usando método HP6890, onde a temperatura inicial de $200{ }^{\circ} \mathrm{C}$ atingindo um máximo de $325^{\circ} \mathrm{C}$ com tempo de corrida de 55,0 minutos. A seringa utilizada foi de $10,0 \mu \mathrm{L}$ e o volume de injeção foi de $1,0 \mu \mathrm{L}$. A detecção foi realizada através de espectrometria de massas.

\section{Formulação dos comprimidos}

Para avaliação do desempenho dos novos nanomateriais como excipientes, foi elaborada uma formulação-base 
Tabela 1. Coluna/CG.

\begin{tabular}{cc}
\hline \multicolumn{2}{c}{ Coluna capilar } \\
\hline Comprimento & $30,0 \mathrm{~m}$ \\
Diâmetro & $250,0 \mu \mathrm{m}$ \\
Espessura do filme & $0,25 \mu \mathrm{m}$ \\
Modo & Fluxo constante \\
Fluxo inicial & $0,8 \mathrm{~mL} / \mathrm{min}$ \\
Pressão inicial & $12,8 \mathrm{psi}$ \\
Velocidade média & $35 \mathrm{~cm} / \mathrm{seg}$ \\
Entrada & Frontal \\
Saída & MSD \\
Pressão saída & Vácuo \\
\hline
\end{tabular}

Tabela 2. Injetor/CG.

\begin{tabular}{cc}
\hline \multicolumn{2}{c}{ Injetor split/splitless } \\
\hline Modo & Split \\
Temperatura inicial & $270{ }^{\circ} \mathrm{C}$ \\
Pressão & $12,8 \mathrm{psi}$ \\
Razão split & $20: 01$ \\
Fluxo split & $16,0 \mathrm{~mL} / \mathrm{min}$ \\
Fluxo total & $19,0 \mathrm{~mL} / \mathrm{min}$ \\
Gás & $\mathrm{Hélio}$ \\
Fluxo & $20,0 \mathrm{~mL} / \mathrm{min}$ \\
Tempo & $2,0 \mathrm{~min}$ \\
\hline
\end{tabular}

com dapsona $100 \mathrm{mg}$, um ativo de classe II na Classificação Biofarmacêutica $^{[12]}$, utilizando-se $30 \%$ em peso das matrizes preparadas, $1,5 \%$ de estearato de magnésio e quantidade de lactose necessária para se obter $100 \%$ do peso. Em todos os casos, os comprimidos provenientes da formulação foram preparados por compressão direta em compressora excêntrica da marca Stockes. Os comprimidos foram avaliados pelos testes preconizados pela Farmacopéia Americana $30^{[9]}$.

\section{Estudos de liberação in vitro}

Para os ensaios de dissolução foi utilizado o aparato II (pá) e estes conduzidos em temperatura de $37^{\circ} \mathrm{C}$ em $900 \mathrm{~mL}$ de meio de dissolução. A intervalos regulares de tempo, alíquotas de $5 \mathrm{~mL}$ de volume foram coletadas e quantificadas, em comprimento de onda apropriado, por espectrofotômetro Perkin-Elmer 734 UV-Vis. Os experimentos foram realizados em triplicata. Os resultados encontrados foram tratados matematicamente de acordo com a cinética de liberação dos ativos de suas respectivas matrizes, de forma que:

$$
\text { Ordem zero: } \% \mathrm{D}=\mathrm{a}_{1}+\mathrm{b}_{1} \cdot \mathrm{t}
$$

$$
\text { Primeira ordem: } \ln (100-\% \mathrm{D})=\mathrm{a}_{2}-\mathrm{b}_{2} \cdot \mathrm{t}
$$

$$
\text { Higuchi: } \% \mathrm{D}=\mathrm{a}_{3}-\mathrm{b}_{3} \cdot \mathrm{t}
$$

onde, \%D, é o valor percentual de ativo liberado; t, é o tempo; e $a_{n}$ e $b_{n}$, representam os coeficientes linear e angular, respectivamente. Foi utilizado o pacote estatístico Statistica para avaliar a influência de diferentes parâmetros na liberação do ativo.

\section{Resultados e Discussão}

\section{Caracterização do viscogel B8}

\section{Análise por infravermelho (FTIR)}

$\mathrm{O}$ espectro da bentonita intercalada com sal de octadecilamina apresenta algumas diferenças quando comparado com o espectro da bentonita sódica. A banda em $3446 \mathrm{~cm}^{-1}$ mostra-se bem menos intensa, devido à ausência quase total de água entre suas lamelas. As bandas correspondentes à deformação axial de $\mathrm{C}-\mathrm{H}\left(2962 \mathrm{~cm}^{-1}\right), v_{\mathrm{as}} \mathrm{CH}_{3}\left(2872 \mathrm{~cm}^{-1}\right)$, $v_{\mathrm{s}} \mathrm{CH}_{3}\left(2924 \mathrm{~cm}^{-1}\right), v_{\text {as }} \mathrm{CH}_{2}\left(2853 \mathrm{~cm}^{-1}\right)$ decorrentes da presença da cadeia alquílica C18 podem ser observadas. A banda correspondente à deformação angular simétrica no plano de N-H é observada na região de $1650-1580 \mathrm{~cm}^{-1}$ com intensidade de média a forte ${ }^{[13]}$.

\section{Difração de raio X (DRX)}

O difratograma do viscogel B8 mostrou que a distância interlamelar desta bentonita modificada organicamente é superior ao da bentonita sódica assumindo um valor de espaçamento interlamelar igual a $26,50 \AA^{[8]}$ de acordo com a equação de $\mathrm{Bragg}^{[7]}$. Isto evidencia o aumento do espaço interlamelar característico de argilas intercaladas.

Análise termogravimétrica (TGA)/análise calorimétrica de varredura (DSC)

$\mathrm{O}$ viscogel $\mathrm{B} 8$ apresenta três etapas de perda de massa onde a primeira etapa ocorre entre 25 e $120^{\circ} \mathrm{C}$ e se refere à evaporação de água e as demais perdas, entre 120 e $520^{\circ} \mathrm{C}$, e 520 e $750{ }^{\circ} \mathrm{C}$, referentes à degradação do sal de octadecilamina, sendo estas suas principais características ${ }^{[8]}$. A análise por DSC evidencia endoterma nas mesmas faixas de temperatura que as associadas a degradação do sal de octadecilamina, especificamente entre 520 e $750{ }^{\circ} \mathrm{C}$, onde não se observa a interferência da perda de água na análise.

\section{Teste de determinação de toxicidade a $10 \mathrm{~g} / \mathrm{kg}$ de peso corpóreo}

Nenhuma morte foi observada nos 14 dias de observação dos camundongos na dose fixa de $10 \mathrm{~g} / \mathrm{kg}$ de peso corpóreo. De acordo com o Sistema de Classificação Integrada Conciliado para Saúde Humana Relacionado à Exposição de Risco a Substâncias e Misturas Químicas ${ }^{[14]}$, o viscogel B8 é uma substância que não oferece riscos de toxicidade na dose utilizada no teste, visto que esse valor é superior ao da dose de classe 5 , que é de $5.000 \mathrm{mg} / \mathrm{kg}$.

\section{Nanocompósito viscogel B8-MEG}

O nanocompósito foi obtido pela técnica de evaporação do solvente (diclorometano), a temperatura ambien- 
te e sob refluxo. Apesar de este solvente não ser o que proporcionaria o maior intumescimento do viscogel $\mathrm{B} 8, \mathrm{o}$ mesmo foi eleito visto sua facilidade de remoção e menor toxicidade comparativa. Nenhuma diferença significativa de rendimento foi detectada entre os diferentes valores de tempo e temperatura de intercalação utilizados, tendo os valores variados de 22 a $27 \%$ em massa de rendimento, compatíveis com os valores descritos em literatura para processos de intercalação similares onde se utilizou o poli(óxido de etileno) como material intercalante ${ }^{[15]}$. Desta forma, optou-se em trabalhar com a agitação por 12 horas a temperatura ambiente ( $25 \%$ de rendimento de inclusão), condições onde se obtêm menores tempo reacional e gasto de energia, viabilizando um mais fácil escalonamento do processo.

Nesta intercalação, buscou-se inserir uma matriz de liberação prolongada de caráter lipofílico no interior do viscogel B8, levando em consideração que combinação sinérgica das propriedades físico-químicas destes dois materiais, otimizaria sua utilização como matriz de liberação prolongada. Originaria-se, assim, um nanocompósito com características de intumescimento e cinética de liberação de ativo adequáveis ao perfil zero ordem, decorrente da combinação das propriedades dos materiais de partida.

\section{Caracterização}

\section{Análise por infravermelho (FTIR)}

Por esta análise não pôde ser visto deslocamento das bandas principais dos materiais de partida, não se prestando, portanto, para caracterizar de forma inequívoca o novo material formado. O que se verifica na análise do espectro do nanocompósito viscogel B8-MEG é resultado do somatório dos espectros do viscogel B8 e MEG isoladamente.

\section{Difração de raio X (DRX)}

O MEG provocou um deslocamento interlamelar no vis-

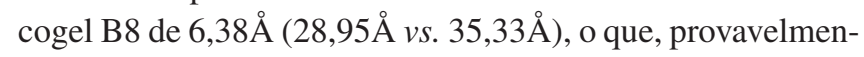
te, indica que o MEG ocupou parte do espaço interlamelar, provocando seu aumento (Figura 1).

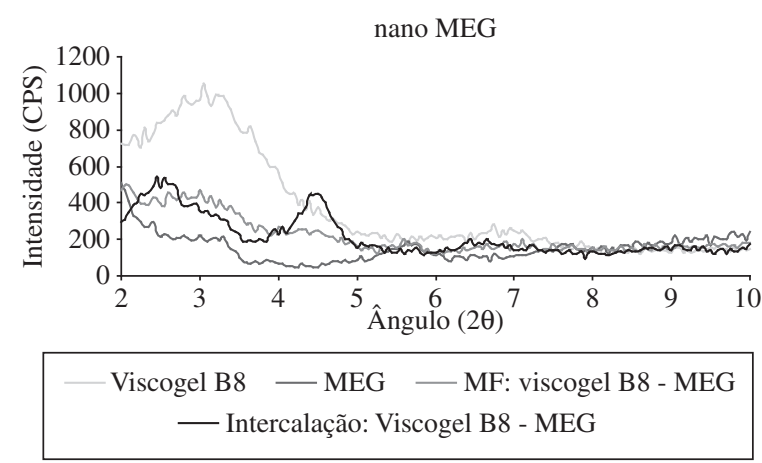

Figura 1. Difratograma da intercalação viscogel B8-MEG.

\section{Análise termogravimétrica (TGA)/análise calorimé- trica de varredura (DSC)}

Os resultados de TGA/DSC demonstram ter havido um deslocamento da endoterma referente ao MEG, corroborando a possibilidade de formação do novo material (Figura 2).

A fim de se pesquisar a manutenção ou não do íon alquilamônio no interior das lamelas da argila e o teor de intercalação foi realizada análise cromatográfica do sobrenadante. O cromatograma obtido para o produto de intercalação revelou ausência de octadecilamina (tempo de retenção $=3,81$ minutos) (Figura 3 ). Deste modo, foi descartada a possibilidade de competição entre os grupos do MEG e octadecilamina. Além disso, pôde ser confirmado o percentual de $25 \%$ de intercalação de MEG no interior do viscogel B8.

Desta forma, foi evidenciada a formação de um novo nanomaterial em rendimentos consideráveis e de aplicação óbvia pelas razões supracitadas em tecnologia farmacêutica como matriz de liberação prolongada. Sua similaridade à cera de carnaúba, que tem mais de $85 \%$ de sua composição formada por ésteres de ácidos graxos ${ }^{[16]}$, ainda aponta para diversas outras aplicações deste material em diferentes áreas tecnológicas.

\section{Nanocompósito viscogel B8-PEG 6000}

Visto que o PEG apresenta uma solubilidade em torno de $4,0 \% \mathrm{em}$ tolueno, e que neste solvente foi obtido um maior inchamento da argila, este foi considerado como solvente de escolha para a reação de intercalação. O polímero é encontrado com peso molecular 4000, 6000, 8000, 12000 e 20000, sendo o 6000 o mais utilizado em aplicações tecnológicas ${ }^{[17]}$.

\section{Análise por infravermelho (FTIR)}

O espectro de FTIR não apontou qualquer diferenciação entre o nanocompósito e a mistura física, sendo, portanto, inconclusivo.

\section{Difração de raio $X$ (DRX)}

Ao se analisar os difratogramas obtidos, pode ser verificado que o melhor tempo de reação sob refluxo foi de 72 horas, já que esta condição apresentou um maior deslocamento do sinal referente ao espaçamento das lamelas do que as demais (Figura 4), correspondendo a um valor de espaçamento interlamelar de 31,00Å.

$\mathrm{O}$ mesmo foi observado para as reações conduzidas a temperatura ambiente (Figura 5), com 72 horas reacionais é encontrado um valor de espaçamento de $31,89 \AA$, resultado que justifica a escolha por este sistema.

O espaçamento aparentemente pequeno pode não necessariamente estar indicando um baixo percentual de intercalação. A variação poderia ser função de uma alteração conformacional do íon alquilamônio, como já descrito por Lebaron e colaboradores ${ }^{[6]}$. Para corroborar este resultado, foi estudado, por difração de raio $\mathrm{X}$, o efeito do solvente 
Module: TGA/SDTA851e/LF1100/MT5/440, 16.10.2001 18:00:51 Method: BL_RP_Dyn25...800@20 - N2 = 50 mL->Alox_70 $\mu \mathrm{L}$ $25.0-800.0{ }^{\circ} \mathrm{C} 20.00{ }^{\circ} \mathrm{C} / \mathrm{min} \quad \mathrm{N} 250.0 \mathrm{~mL} / \mathrm{min}$
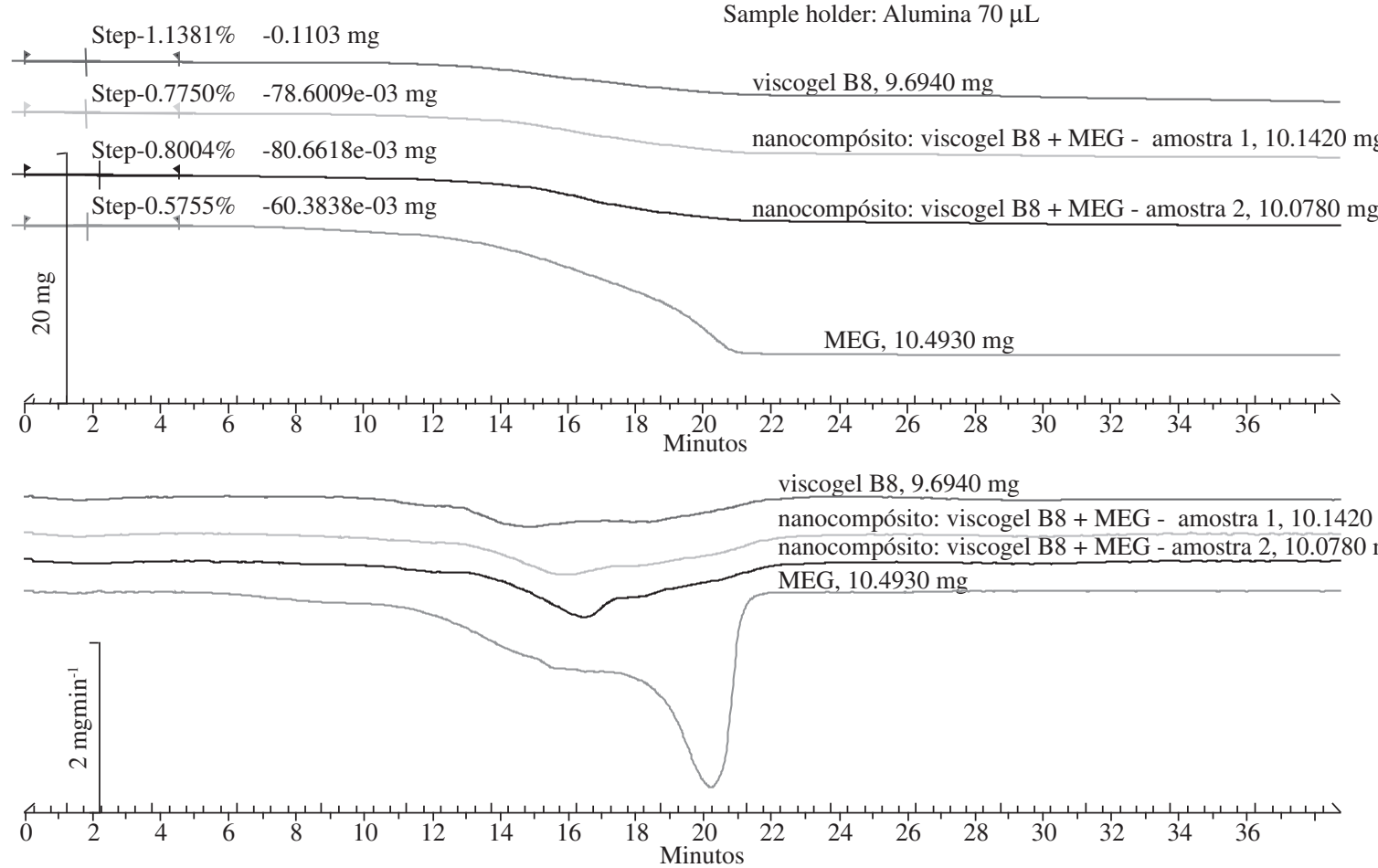

Figura 2. TGA/DSC relacionados aos materiais: (---) viscogel B8; (---) viscogel B8-MEG (1); (---) viscogel B8-MEG (2); (---) MEG.

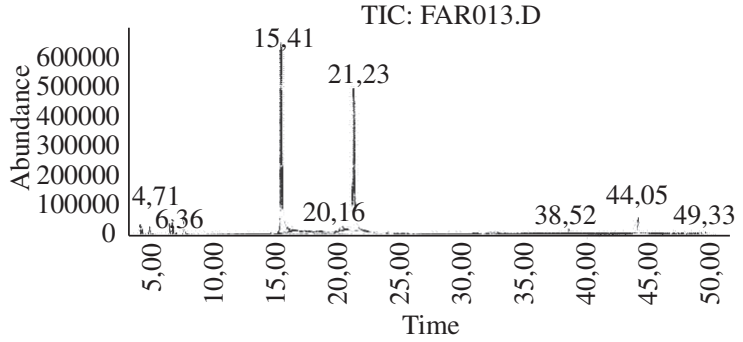

\begin{tabular}{cccc}
\hline Retention time & Área & Área \% & Ratio \% \\
\hline $\begin{array}{c}\text { Total íon } \\
\text { chromatogram }\end{array}$ & & & \\
4.020 & 634190 & 0.516 & 1.136 \\
4.187 & 718133 & 0.584 & 1.286 \\
4.712 & 3066419 & 2.493 & 5.493 \\
6.367 & 1493891 & 1.215 & 2.676 \\
6.620 & 1726063 & 1.404 & 3.092 \\
7.506 & 2957137 & 2.405 & 5.297 \\
15.406 & 55825196 & 45.393 & 100.000 \\
20.156 & 2540853 & 2.066 & 4.551 \\
21.233 & 46728427 & 37.996 & 83.705 \\
38.516 & 1085730 & 0.883 & 1.945 \\
44.049 & 3355538 & 2.728 & 6.011 \\
49.328 & 2849713 & 2.317 & 5.105 \\
\hline
\end{tabular}

Figura 3. Cromatograma do sobrenadante obtido da reação viscogel B8-MEG.

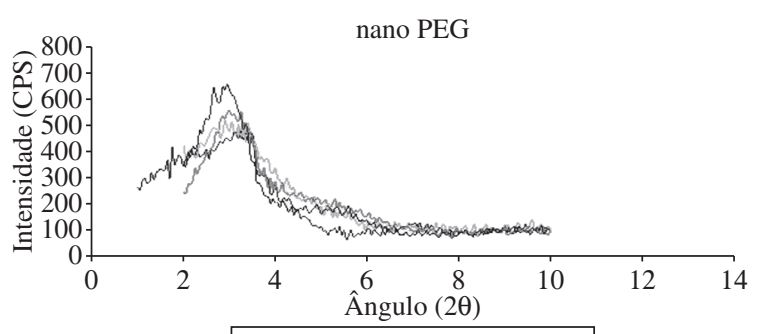

\begin{tabular}{|ll|}
\hline- Viscogel B8 & -48 horas \\
-24 horas & -72 horas
\end{tabular}

Figura 4. Difratograma das reações viscogel B8-PEG sob refluxo.

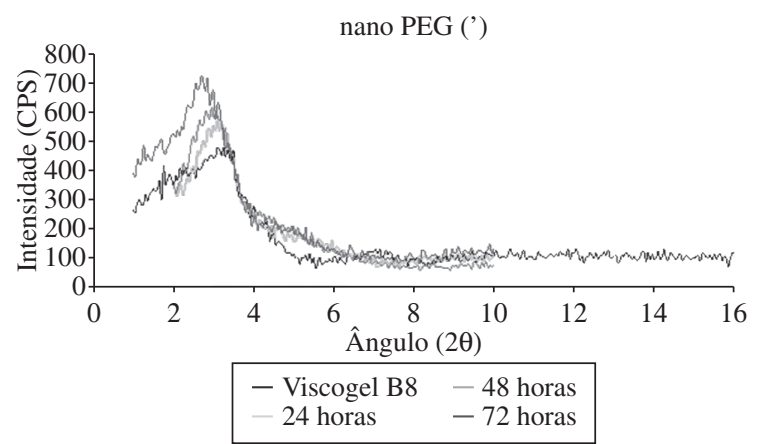

Figura 5. Difratograma das reações viscogel B8-PEG a temperatura ambiente. 
Tabela 3. Espaçamento interlamelar - avaliação do efeito do solvente.

\begin{tabular}{ccc}
\hline $\begin{array}{c}\text { Solvente/tempo } \\
\text { (horas) }\end{array}$ & Ângulo 2 $\theta$ & $\begin{array}{c}\text { Tamanho de } \\
\text { lamela }(\AA)\end{array}$ \\
\hline- & 3,30 & 26,8 \\
Dicloroetano/72 & 3,85 & 22,9 \\
Diclorometano/72 & 4,05 & 21,8 \\
Tolueno/72 & 4,05 & 21,8 \\
\hline
\end{tabular}

na variação do espaçamento interlamelar ao se refluxar a argila com diferentes solventes por 72 horas. Os resultados obtidos (Tabela 3) confirmam esta variação e a diferença na variação em função do solvente escolhido.

Análise termogravimétrica (TGA)/análise calorimétrica de varredura (DSC)

Foi feita a análise termogravimétrica da melhor condição eleita ( 72 horas em temperatura ambiente) e os resultados podem ser conferidos pela Figura 6. É verificada uma melhora da estabilidade térmica do nanocompósito em relação à mistura física, indicando haver uma variação próxima a $10{ }^{\circ} \mathrm{C}$ em relação ao início do processo de degradação da mistura física para o nanocompósito. Este aumento na estabilidade térmica do nanocompósito é compatível com o verificado por Loyens e colaboradores (2005) no preparo de argilas modificadas com oligômeros de PEG por fusão, assim como o valor percentual de polímero intercalado, próximo de $38,9 \%{ }^{[18]}$.

Da análise de DSC não são observadas importantes variações dos valores de $\mathrm{T}_{\mathrm{m}}$ dos nanocompósitos para a mistura física.

\section{Nanocompósito viscogel B8-PVP K-30}

A reação entre a organoargila e a PVP se passa em um tempo reacional bem inferior (em 45 minutos a temperatura ambiente) em comparação aos outros polímeros estudados, provavelmente em função de sua estrutura amídica (Figura 7a). Como pode ser observado pelo termograma a seguir (Figura 8), nota-se a ausência da última etapa de perda de massa (entre 520 e $750{ }^{\circ} \mathrm{C}$ ), o que demonstra que a PVP seria não somente capaz de estabilizar as lamelas negativamente carregadas do viscogel B8, mas, inclusive, de competir com o íon alquilamônio, deslocando-o do espaço interlamelar. Desta forma, através da análise das propriedades físicoquímicas dos polímeros estudados, avaliando-se as possíveis interações que ocorreriam entre os dipolos presentes na PVP e aqueles presentes no interior da cavidade interlamelar da

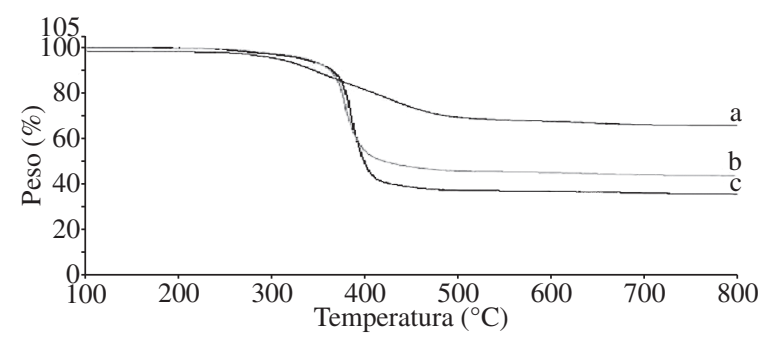

Figura 6. Curvas de termogravimetria para: a) viscogel B8; b) mistura física de viscogel B8-PEG; e c) reação viscogel B8-PEG.

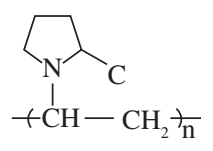

(a)

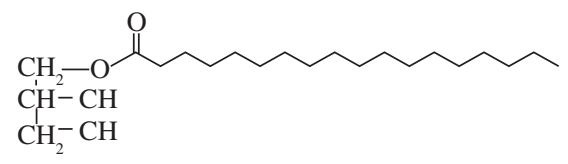

(b)<smiles>CCOCCOCCO</smiles>

(c)

Figura 7. Estruturas químicas de: a) PVP; b) MEG; e c) PEG.

viscogel B8, pode-se justificar a PVP ser intercalada mais rapidamente que MEG (Figura 7b), o qual tem sua intercalação facilitada pelo maior espaço interlamelar obtido pela inserção do íon alquilamônio, além de apresentar massa molar menor que os demais polímeros já intercalados com esta argila, parâmetro que facilita o processo. Com esta macromolécula, não se observa a remoção dos íons alquilamônio, conforme análise por CG. Já o PEG, por não apresentar lipofilicidade, tampouco afinidade pelas lamelas da argila (Figura 7c), resulta em menores rendimentos de intercalação necessidade de condições mais enérgicas de processamento.

\section{Avaliação dos novos nanocompósitos como matrizes de} liberação prolongada

\section{Nanocompósito viscogel B8-MEG}

Em um teste preliminar de intumescimento este nanocompósito mostrou uma capacidade de absorção de água bastante reduzida quando comparado à hidroxipropilmetilcelulose (HPMC), uma matriz hidrofílica de elevada capacidade de intumescimento e reconhecida eficiência retardante ${ }^{[19]}$. O resultado descarta seu uso como matriz de liberação prolongada, mas não sua utilização como outro tipo de excipiente farmacêutico. Quanto à compactação, com respeito à possibilidade de se utilizar este material em compressão direta, verifica-se um valor de dureza média em torno de 8,5-9,0 $\mathrm{KgF}$ (após compressão de $600 \mathrm{mg}$ por 2 minutos em 6 toneladas), apontando para esta possibilidade.

\section{Nanocompósito viscogel B8-PEG 6000}

Para tal, foi estudada a liberação in vitro de dapsona (fármaco-modelo), o qual mostraria vantagens quando veiculado em um sistema de liberação prolongada, utilizando-se o suco gástrico simulado (SGS) como meio de dissolução, primeiro compartimento anatômico onde se inicia a dissolução do ativo, completando-se a formulação-base com a inserção do nanocompósito polimérico em questão. Como pode ser observado na Figura 9, é notada uma capacidade retardante superior para o nanocompósito em comparação tanto à sua 

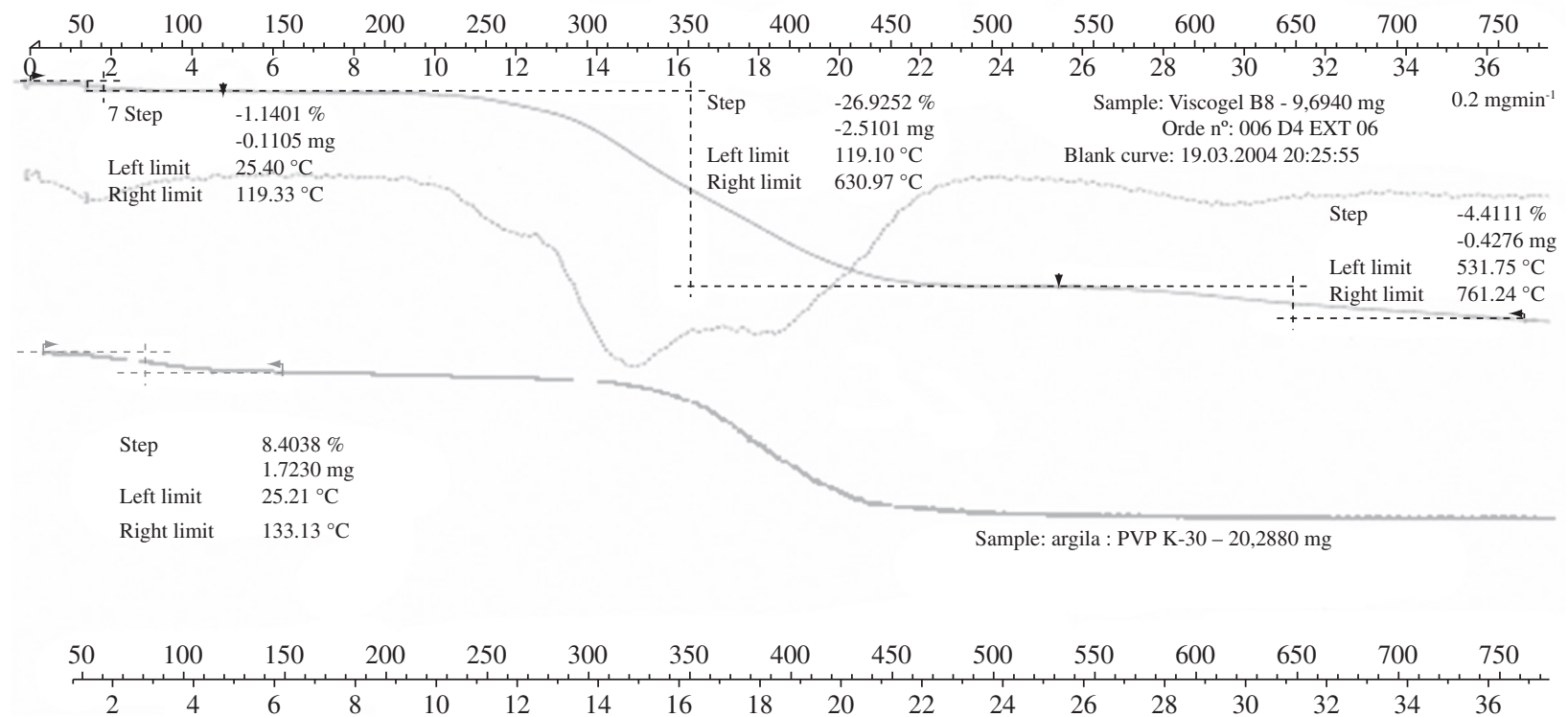

Figura 8. Termogramas de (---) viscogel B8 e (---) nanocompósito viscogel B8-PVP K-30.

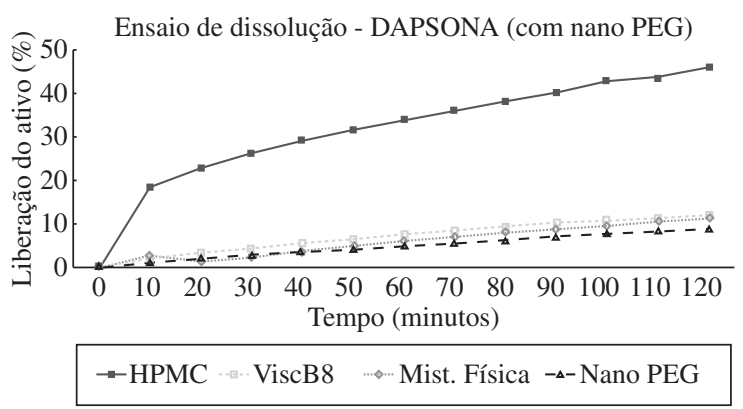

Figura 9. Perfil de dissolução em SGS do nanocompósito viscogel B8-PEG 6000, da mistura física, viscogel puro e HPMC.

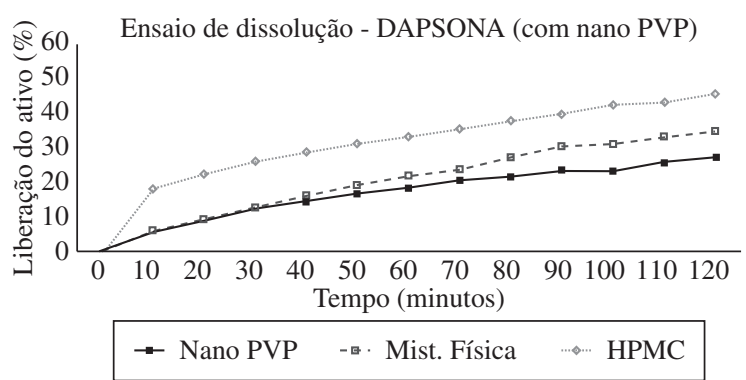

Figura 10. Perfil de dissolução em SGS do nanocompósito viscogel B8-PVP K-30, da mistura física e HPMC.

mistura física $(3,2 \%)$ quanto ao viscogel $\mathrm{B} 8$ isoladamente $(4,0 \%)$ e à HPMC $(37,8 \%)$.

\section{Nanocompósito viscogel B8-PVP K-30}

O desempenho do PEG como matriz retardante foi, ainda, superior ao da PVP K-30, a qual liberou, nas mesmas condições, mais dapsona $(28,7 \%$ vs. $8,4 \%$, respectivamente) durante o mesmo tempo de observação (Figura 10). Todavia o resultado não a descarta como matriz retardante, já que se mostrou mais eficiente que a HPMC.

\section{Conclusões}

Por meio deste estudo foi possível evidenciar a formação, por solução, de nanocompósitos polímero - silicato lamelar com MEG e PEG 6000. Os ensaios de dissolução in vitro, tanto para os nanocompósitos de PEG quanto os obtidos da reação de PVP K-30 com o viscogel B8, demonstram ser estes novos excipientes potenciais matrizes controladoras da cinética de liberação de fármacos.

\section{Agradecimentos}

Ao apoio financeiro fornecido pelo CNPq e CAPES.

\section{Referências Bibliográficas}

1. Minatech Information Day. Conference on Micro and Nano Technologies. A World Wide view. AIRI - Associazione Italiana per la Ricerca Industriale. European Reports on Technologies, Italy (2001).

2. Goddard III, A. W. - "Nanoscale Theory and Simulation: a critical driver for and a critical challenge to convencional nanotechnology". (1998). Disponível em: $<$ http://www.wtec.org/loyola/nano/US.Review/09_07. htm>. Acesso em 28 jun. 2007.

3. White, J. L. \& Hem, S. L. - "Ind. Enginee. Chem. Prod. Res. Develop.” 22 (4), p.665-671 (1983).

4. Erbsloh, G. - "The Clay Bentonite. A fascinanting natural substance and its application in beverage treat- 
ment”. (2004). Disponível em: <www.swiftco.com.au/ swift/pdf/bentonite.pdf> Acesso em 05 dez. 2006.

5. Carrado, K. A. - “Appl. Clay Sci.”, 17, p.1-23 (2000).

6. Lebaron, P. C.; Wang, Z. \& Pinnavaia, T. J. - "Appl. Clay Sci.", 15, p.11-29 (1999).

7. Kornmann, X. (2000). Disponível em: <http://www. mb.luth.se/a_mpp_staff/Xavier.Kornmann/introduction.pdf $>$. Acesso em: 18 dez. 2006.

8. Dornelas, C. B. - "Preparação e caracterização de novos nanocompósitos de PVP - montmorilonita organofílica como excipiente farmacêutico", Dissertação de Mestrado, Universidade Federal do Rio de Janeiro, Brasil (2006).

9. UNITED STATES PHARMACOPEIA (USP). 30 ed. Rockville: U.S.P. Convention (2007).

10. Burgentzlé, D.; Duchet, J.; Gérard, J. F.; Jupin, A. \& Fillon, B. - "J. Colloid Interface Sci", 278, p.26-39 (2004).

11. FARMACOPÉIA BRASILEIRA. 4 ed. São Paulo: Atheneu (1988b).

12. Amidon, G. L.; Lennernäs, H.; Shah, V. P. \& Crison, J. R. - "Pharm. Res.", 12, (3), p.413-420 (1995).
13. Soares, K. C. C. - "Avaliação do uso de bentonita na preparação de nanosistemas com atividade biológica", Dissertação de Mestrado, Universidade Federal do Rio de Janeiro, Brasil (2003).

14. Grande, L. F. - "Estudios de Bioética", Dykinson, Madrid (1997).

15. Esteves, A. C. C.; Barros-Timmons, A. \& Trindade, T. "Quim. Nova", 27, (5), p.798-806 (2004).

16. Jacob, M. T. (2006). Disponível em: <http://www.fnt. org.br/artigos.php?idpessoa=20>. Acesso em: $20 \mathrm{fev}$. (2008).

17. Perez, S. C. J. G. A.; Fanti, S. C. \& Casali, C. A. - "Bragantia", Campinas, 60, (3), p.155-166 (2001).

18. Loyens, W.; Jannasch, P. \& Maurer, F. H. J. - Polymer, 46, p.915-928 (2005a).

19. Lopes, C. M.; Lobo, J. M. S. \& Costa, C. - "Ver. Brás. De Ciências Farmacêuticas”, 41, (2), abr/jun (2005).

Enviado: 06/08/07

Reenviado: 05/03/08

Aceito: $14 / 03 / 08$ 